\title{
Non-Binary Diameter Perfect Constant-Weight Codes
}

\author{
Tuvi ETZION*
}

September 28, 2021

\begin{abstract}
Diameter perfect codes form a natural generalization for perfect codes. They are based on the code-anticode bound which generalizes the sphere-packing bound. The code-anticode bound was proved by Delsarte for distance-regular graphs and it holds for some other metrics too. In this paper we prove the bound for non-binary constantweight codes with the Hamming metric and characterize the diameter perfect codes and the maximum size anticodes for these codes. We distinguish between six families of non-binary diameter constant-weight codes and four families of maximum size nonbinary constant-weight anticodes. Each one of these families of diameter perfect codes raises some different questions. We consider some of these questions and leave lot of ground for further research. Finally, as a consequence, some $t$-intersecting families related to the well-known Erdös-Ko-Rado theorem, are constructed.
\end{abstract}

\footnotetext{
${ }^{*}$ Department of Computer Science, Technion, Haifa 3200003, Israel, e-mail: etzion@cs.technion. ac.il.
} 


\section{Introduction}

Perfect codes are one of the most fascinating structures in coding theory. These codes meet the well-known sphere-packing bound. When the size of a ball does not depend on its center, these codes are the largest ones for their length and minimum distance. They are defined for variety of metrics in coding theory. For most such metrics other bounds which improve on the sphere-packing bound, were developed. One of these bounds is the code-anticode bound which was first introduced by Delsarte in his seminal work [12]. This bound is a direct generalization of the sphere-packing bound. For this bound the concept of anticode is required. An anticode $\mathcal{A}$ with diameter $D$ in a space $\mathcal{V}$ is a subset of $\mathcal{V}$, with a metric, such that the maximum distance between elements in $\mathcal{A}$ is $D$. Delsarte [12] proved the code-anticode bound for distance-regular graphs. The bound was re-introduced and generalized by Ahlswede, Aydinian, and Khachatrian [1] who called any code which meets this bound, a D-diameter perfect code. To apply the code-anticode bound, one must find the size of the maximum size anticode for the related space, metric, and parameters. This diametric problem in some schemes, such as the Hamming scheme and the Johnson scheme was discussed in [1] and shown to be closely related to intersection problems for systems of finite sets which go back to Erdös-Ko-Rado theorem (see [2, 3, 14] and references therein).

The proof of the code-anticode bound which was introduced in [1] required a space and metric associated with a graph which admits a transitive group of automorphisms. The proof in [1] was demonstrated on the Johnson graph. Nevertheless, no general proof was given for the theorem for the family of graphs which admit a transitive group of automorphisms. They have considered the related codes and anticodes in the Hamming scheme, the Johnson scheme, and the Grassmann scheme. Other metrics, which are not associated with distance-regular graphs, for which the proof was generalized, are the $\ell_{\infty}$ metric on the set of permutations $S_{n}$ [33], the Lee metric in $\mathbb{Z}_{m}^{n}$ [17] and the Kendall $\tau$-metric on the set of permutations $S_{n}$ [6]. The bound was also considered for the rank-distance on Ferrers diagrams in [18]. For each one of these spaces and metrics some diameter perfect codes were introduced in these papers.

In this paper we further expand our knowledge on diameter perfect codes and optimal anticodes. We consider the set of all words of length $n$ with weight $w$ over an alphabet $Q$ with $q$ symbols, $q>2$, where the metric used is the Hamming distance. The graph (whose vertices are the space) associated with these words and metric will be denoted by $\mathrm{J}_{q}(n, w)$. Its set of vertices consists of all the words of length $n$ and weight $w$ over an alphabet $Q$ of size $q$, say $\mathbb{Z}_{q}$. Two vertices are connected by an undirected edge if the Hamming distance of their associated words is one. Hence, when $q>2$ the related graph is not connected unless $w=n$. Therefore, the graph is not distance-regular, unless $w=n$, which implies that the direct arguments in [1, 12] do not apply. It should be noted that the graph is a union of disjoint distance-regular graphs, each one is formed from codewords which have the same support. This graph was considered before when $q=3$ and $w=n-1$ in [24, 25] who provided some arguments for the code-anticode bound for this family of codes. Nevertheless, similarly to the proof in [1], the proof in [24, 25] is not complete. In [24] it is proved that there is only one such diameter perfect code with minimum distance 4 , based on the nonexistence of related perfect colorings [20]. There is also a construction in [24] of diameter perfect codes with minimum distance 5. In [25] there is a proof that a diameter perfect code with minimum 
distance 5 and length 16 does not exists. A diameter perfect code with minimum distance 5 and length 64 is derived from a construction in [5]. In this paper, we provide a general proof of the code-anticode bound for $\mathrm{J}_{q}(n, w), q>2$. We distinguish between six families of diameter perfect codes in $\mathrm{J}_{q}(n, w)$ and four families of maximum size anticodes associated with them. It should be noted that each family of non-binary diameter perfect constantweight codes is a family of maximum size constant-weight codes with the related parameters (the length of the codewords will be denoted by $n$, their weight by $w$, the minimum distance of the code by $d$, and the alphabet size by $q$ ). This problem of finding the maximum size of such codes was extensively studied, e.g. [8, 9, 36] and references therein.

The rest of this paper is organized as follows. In Section 2, the sphere-packing bound, the code-anticode bound, and its generalization presented in [1], are introduced. The local inequality lemma from which the code-anticode bound can be proved, is also presented in this section. Based on the code-anticode bound the concept of a diameter perfect code, which is a generalization of a perfect code, is defined. Finally, the set of words with weight $w$ over an alphabet with $q$ letters is defined and some preliminaries are given. In Section 3 , the code-anticode bound is proved for the Johnson scheme and it is shown that Steiner systems and complements of Steiner systems are diameter perfect codes in this scheme. In Section 4, non-binary diameter perfect constant-weight codes are discussed. The codeanticode bound is proved for non-binary constant-weight words and six families of diameter perfect codes are characterized. In Section 4.1, non-binary diameter perfect constant-weight codes for which $w=n$ are discussed. These codes are derived from diameter perfect codes in the Hamming scheme. There is a one-to-one correspondence between these two families of codes. In Section 4.2, non-binary perfect constant-weight codes for which the alphabet size is $2^{k}+1$, the length is $n$, and the weight is $w=n-1$ are considered. Most of these codes are over ternary alphabet. In Section 4.3, we define the family of generalized Steiner systems and show that each generalized Steiner system is a diameter perfect constant-weight code. In Section 4.4, the family of MDS constant-weight codes is defined. This family got its name from MDS codes since the minimum weight codewords of any MDS code form such a code. Section 4.5 is devoted to codes for which $d=w+1$. Codes in this family have exactly one codeword for each support which consists of any $w$-subset of the $n$ coordinates. In Section 4.6, the last family called multiple orthogonal arrays constant-weight codes is defined. For this family $d<w$ and each $w$ coordinates are the supports of codewords which form an orthogonal array. In Section 4.7, we compare between the different maximum size anticodes associated with the six families of diameter perfect constant-weight codes. We distinguish between four families of such anticodes. Finally, in Section 5 we summarize our results and suggest a list of open problems for future research.

\section{The Code-Anticode Bound and Preliminaries}

Let $\mathcal{V}$ be a space and $d: \mathcal{V} \times \mathcal{V} \longrightarrow \mathbb{Z}$ be a metric defined on $\mathcal{V}$. In the metric $d$, the $\boldsymbol{b a l l}$, $\mathcal{B}_{e}(x)$ of radius $e$ around $x \in \mathcal{V}$ is the set of elements which are at distance $e$ from $x$, i.e. $\mathcal{B}_{e}(x) \triangleq\{y: d(x, y) \leq e\}$. The metric is called regular if the size of the ball does not depend on its center. In this case the ball of radius $e$ is denoted by $\mathcal{B}(e)$. The well-known sphere-packing bound is introduced in the following theorem. 
Theorem 1. If $\mathcal{C} \subseteq \mathcal{V}$ is a code with minimum distance $2 e+1$, associated with a regular metric, then

$$
|\mathcal{C}| \cdot|\mathcal{B}(e)| \leq|\mathcal{V}|
$$

A code which meets the bound of Theorem 1 is called a perfect code. Theorem 1 is generalized by the code-anticode bound which will be introduced now. The bound is an immediate consequence of the following theorem which was proved by Delsarte [12].

Theorem 2. Let $\mathcal{V}$ be the set of vertices in a distance-regular graph and let $X$ and $Y$ be two set of vertices in $\mathcal{V}$. If the nonzero distances occurring between vertices of $X$ do not occur between vertices of $Y$, then

$$
|X| \cdot|Y| \leq|\mathcal{V}|
$$

Corollary 1. Let $\mathcal{V}$ be the set of vertices in a distance-regular graph and let $X$ and $Y$ be two sets of vertices in $\mathcal{V}$. If the the minimum distance in $X$ is $D+1$ and the maximum distance in $Y$ is $D$, then

$$
|X| \cdot|Y| \leq|\mathcal{V}|
$$

Theorem 2 was proved in [12] for distance-regular graphs using the duality theorem for linear programming. Much simpler proofs to the same bound for distance-regular graphs were given by [13] and [31]. Theorem 2 was generalized by Ahlswede, Aydinian, and Khachatrian [1] with the following lemma which is the key result required for the definition of diameter perfect codes. It has several proofs, depending on the space and the metric being considered. Nevertheless, there are spaces and metrics for which this lemma is not satisfied and hence it cannot be used for those spaces and metrics.

Lemma 1. Let $\mathcal{C}_{\mathcal{D}}$ be a code in a space $\mathcal{V}$ with a metric $d: \mathcal{V} \times \mathcal{V} \longrightarrow \mathbb{Z}$ (satisfying some conditions), where the distances between the codewords in $\mathcal{C}$ are taken from a subset $\mathcal{D}$. Let $\mathcal{A}$ be a subset of $\mathcal{V}$ and let $\mathcal{C}_{\mathcal{D}}^{\prime} \subseteq \mathcal{A}$ be the largest code in $\mathcal{A}$ with distances taken from $\mathcal{D}$. Then

$$
\frac{\left|\mathcal{C}_{\mathcal{D}}\right|}{|\mathcal{V}|} \leq \frac{\left|\mathcal{C}_{\mathcal{D}}^{\prime}\right|}{|\mathcal{A}|}
$$

The conditions required for Lemma 1 can be different depending on the space and the metric. The lemma was proved for several such spaces and metrics, e.g. [6, 17, 18, 33]. Lemma 1 will be referred to as the local inequality lemma and (1) as the local inequality bound. The local inequality lemma implies the code-anticode bound as follows. Choose $\mathcal{A}$ in Lemma 1 to be an anticode with diameter $D$ and choose $\mathcal{C}_{\mathcal{D}}$ to be a code with distances between codewords taken from the subset $\mathcal{D} \triangleq\{i: D+1 \leq i \leq \Delta\}$, for some $\Delta \geq D+1$. Hence, $\mathcal{C}_{\mathcal{D}}^{\prime}$ is a code with exactly one codeword and therefore (11) implies that

$$
\left|\mathcal{C}_{\mathcal{D}}\right| \cdot|\mathcal{A}| \leq|\mathcal{V}|
$$

which will be referred to as the code-anticode bound.

Lemma 1 was proved in [1] only for the Johnson graph $\mathrm{J}(n, w)$, but it was claimed that it holds for any graph which admits a transitive group of automorphism. The proof for the Johnson graph was not trivial and a different proof, based on the transitive operation, should be adapted to different distance-regular graphs. In particular it can be easily adapted to the 
Hamming graph. Distance-regular graphs are associated with association schemes [12] from which the most important one in coding theory is the Hamming scheme associated with the Hamming distance. The following lemma is well-known in the Hamming scheme as well as for any other metric. It can be proved using the triangle inequality.

Lemma 2. A ball with radius e in a metric is an anticode with diameter $2 e$.

Proof. Let $y, z$ be two distinct words in a the ball centered at some word $x$, i.e., $d(y, x) \leq e$ and $d(x, z) \leq e$. Hence, by the triangle inequality,

$$
d(y, z) \leq d(y, x)+d(x, z) \leq 2 e,
$$

which implies that an ball with radius $e$ is an anticode with diameter $2 e$.

A code $\mathcal{C}$ which attains (21) with equality is called a D-diameter perfect code. The following theorem implies a tight connection between perfect codes and diameter perfect codes. It is an immediate consequence from Theorem 1 and Lemma 2.

Theorem 3. If $d: \mathcal{V} \times \mathcal{V} \longrightarrow \mathbb{Z}$ is a regular metric for which the local inequality bound (1) is satisfied, then an e-perfect code in $\mathcal{V}$ is a (2e)-diameter perfect code.

We continue with the spaces and metrics which will be discussed in this paper.

Definition 1. Let $J(n, w)$ denote the set of all $w$-subset of an $n$-set (or equivalently, the set of all the binary words of length $n$ and weight $w$ ), with the Johnson distance (defined as half of the Hamming distance).

Definition 2. Let $J_{q}(n, w), q>2$, denote the set of all the words of length $n$ and weight $w$, over $\mathbb{Z}_{q}$, with the Hamming distance.

Clearly, both $\mathrm{J}(n, w)$ and $\mathrm{J}_{q}(n, w)$ are contained in the Hamming scheme. But, $\mathrm{J}(n, w)$ is a well-known association scheme, while $\mathrm{J}_{q}(n, w)$ does not define an association scheme and its related graph is not connected. But, since both subsets are part of the Hamming scheme, they share the following property of the Hamming scheme implied by Theorem 3 .

Theorem 4. An e-perfect code in $J(n, w)$ is a (2e)-diameter perfect code in $J(n, w)$. If the local inequality lemma holds for $J_{q}(n, w)$, then an e-perfect code in $J_{q}(n, w)$ is a (2e)-diameter perfect code in $J_{q}(n, w)$.

The following definitions will be used in the sequel. For a word $x=\left(x_{1}, x_{2}, \ldots, x_{n}\right)$ over an alphabet $Q$, the support of $x, \operatorname{supp}(x)$, is the subset of nonzero coordinates in $x$, i.e., $\operatorname{supp}(x) \triangleq\left\{i: x_{i} \neq 0,1 \leq i \leq n\right\}$. The weight of $x, \operatorname{wt}(x)$, is the number of nonzero coordinates in $x$, i.e., $\operatorname{wt}(x) \triangleq\left|\left\{i: x_{i} \neq 0,1 \leq i \leq n\right\}\right|=|\operatorname{supp}(x)|$. Let $Q$ denote an alphabet of size $q$ which contains a zero and let $Q^{*} \triangleq Q \backslash\{0\}$.

Definition 3. An $(n, d, w)_{q}$ code is a code of length $n$ over an alphabet with $q>2$ symbols having minimum Hamming distance $d$. Let $A_{q}(n, d, w)$ denote the maximum size of an $(n, d, w)_{q}$ code. 
In the sequel, for some constructions and bounds we will require to use MDS codes and their nonlinear version of orthogonal arrays. An orthogonal array $\mathrm{OA}_{\lambda}(t, n, q)$ is an $\left(\lambda q^{t}\right) \times n$ array $\mathcal{M}$ over an alphabet $Q$ with $q$ symbols such that in each projection of $t$ columns from $\mathcal{M}$ each ordered $t$-tuple of $Q$ appears in exactly $\lambda$ rows. When $\lambda=1$ the orthogonal array is denoted by $\mathrm{OA}(t, n, q)$ and is called an orthogonal array with index unity. If such an orthogonal array with index unity is a linear code, then the code is called a maximum distance separable code (an MDS code in short). This code has length $n$, dimension $t$, and minimum Hamming distance $n-t+1$. An orthogonal array $\mathrm{OA}(t, n, q)$ is also a $(n-t)$-diameter perfect code [1] in the Hamming scheme. The related maximum size anticode of length $n$, diameter $n-t$, and $q^{n-t}$ codewords is defined by

$$
\left.\left\{(a_{1}, a_{2}, \ldots, a_{n-t} \overbrace{0 \cdots \cdots 0}^{t \text { times }})\right): a_{i} \in Q, 1 \leq i \leq n-t\right\} .
$$

There are some well-known bounds on the tradeoff between $t, n$, and $q$ (see for example [30, pp. 11-16]).

Theorem 5. If there exists an $O A(2, n, q)$ then $n \leq q+1$.

Theorem 6. Assume that there exists an $O A(t, n, q)$, where $t \geq 3$ and $q \geq t$.

1. If $q$ is even then $n \leq q+t-1$.

2. If $q$ is odd then $n \leq q+t-2$.

Theorem 7. If there exists an $O A(t, n, q)$, where $q \leq t$, then $n \leq t+1$.

The following result [27, pp. 317-331] is derived from MDS codes.

Theorem 8. If $t \leq n-2$, then there exists an $O A(t, n, q)$ if $n \leq q+1$ for each prime power $q$ and $2 \leq t \leq q-1$. The only exception is when $q$ is even and $t \in\{3, q-1\}$ in which case $n \leq q+2$.

\section{Binary Diameter Perfect Constant-Weight Codes}

Our first step is to prove the local inequality lemma and as a consequence to prove the codeanticode bound. The local inequality lemma for the Johnson scheme was proved in [1] and for completeness the proof is presented here. It will be interesting to look on the difference between the proof of this lemma for the Johnson scheme and its proof for constant-weight codes over a non-binary alphabet (see Lemma 8).

Lemma 3. Let $\mathcal{C}_{\mathcal{D}}$ be a code in $J(n, w)$ with distances between the codewords of $\mathcal{C}_{\mathcal{D}}$ are taken from a subset $\mathcal{D}$. Let $\mathcal{A}$ be a subset of $J(n, w)$ and let $\mathcal{C}_{\mathcal{D}}^{\prime} \subseteq \mathcal{A}$ be the largest code in $\mathcal{A}$ with distances taken from $\mathcal{D}$. Then

$$
\frac{\left|\mathcal{C}_{\mathcal{D}}\right|}{\left(\begin{array}{c}
n \\
w
\end{array}\right)} \leq \frac{\left|\mathcal{C}_{\mathcal{D}}^{\prime}\right|}{|\mathcal{A}|} .
$$


Proof. Consider the set of pairs

$$
\mathcal{P}=\left\{(c, \pi): c \in \mathcal{C}_{\mathcal{D}}, \pi \in S_{n}, \pi(c) \in \mathcal{A}\right\}
$$

For a fixed $c \in \mathcal{C}_{\mathcal{D}}$ and a fixed $a \in \mathcal{A}$ there are exactly $w !(n-w)$ ! choices for a permutation $\pi$, such that $a=\pi(c)$. Hence, the number of pairs in $\mathcal{P}$ equals to $\left|\mathcal{C}_{\mathcal{D}}\right| \cdot|\mathcal{A}| \cdot w ! \cdot(n-w)$ !.

Note, that for each permutation $\pi$ and two elements $x, y \in \mathrm{J}(n, w)$, we have that $d(\pi(x), \pi(y))=d(x, y)$. It implies that a fixed permutation $\pi \in S_{n}$ can transfer the elements of $\mathcal{C}_{\mathcal{D}}$ into at most $\left|\mathcal{C}_{\mathcal{D}}^{\prime}\right|$ elements of $\mathcal{A}$. Therefore, each permutation $\pi$ contributes at most $\left|\mathcal{C}_{\mathcal{D}}^{\prime}\right|$ pairs to $\mathcal{P}$, and hence the number of pairs in $\mathcal{P}$ is at most $\left|\mathcal{C}_{\mathcal{D}}^{\prime}\right| \cdot n$ ! which implies that

$$
\left|\mathcal{C}_{\mathcal{D}}\right| \cdot|\mathcal{A}| \cdot w ! \cdot(n-w) ! \leq\left|\mathcal{C}_{\mathcal{D}}^{\prime}\right| \cdot n !
$$

and the claim of the lemma follows.

Lemma 3 implies that the code-anticode bound is satisfied for the Johnson scheme.

Corollary 2. If $\mathcal{C}$ is a code in $J(n, w)$ with minimum Johnson distance $D+1$ and $\mathcal{A}$ is an anticode in $J(n, w)$ with maximum Johnson distance $D$, then

$$
|\mathcal{C}| \cdot|\mathcal{A}| \leq\left(\begin{array}{l}
n \\
w
\end{array}\right)
$$

The following lemma is readily verified.

Lemma 4. The set $\mathcal{A}(n, w, t)$, where $0 \leq t \leq w \leq \frac{n}{2}$, defined by $\mathcal{A}(n, w, t) \triangleq\left\{(\overbrace{1 \cdots \cdots 1}^{t \text { times }}, a_{1}, \ldots, a_{n-t}): a_{j} \in\{0,1\}, 1 \leq j \leq n-t, w t\left(a_{1}, \ldots, a_{n-t}\right)=w-t\right\}$, is an anticode in $J(n, w)$ whose diameter is $w-t$ and its size is $\left(\begin{array}{l}n-t \\ w-t\end{array}\right)$.

For a binary code $\mathcal{C}$ of length $n$, the complement of $\mathcal{C}, \overline{\mathcal{C}}$, is defined by

$$
\overline{\mathcal{C}} \triangleq\left\{\left(\bar{x}_{1}, \bar{x}_{2}, \ldots, \bar{x}_{n}\right):\left(x_{1}, x_{2}, \ldots, x_{n}\right) \in \mathcal{C}\right\}
$$

where $\bar{b}$ is the binary complement of $b \in\{0,1\}$.

Lemma 5. The set $\overline{\mathcal{A}}(n, w, t)$, where $0 \leq t \leq w \leq \frac{n}{2}$, defined by

$$
\overline{\mathcal{A}}(n, w, t) \triangleq\left\{(\overbrace{0 \cdots \cdots 0}^{t \text { times }}, a_{1}, \ldots, a_{n-t}): a_{j} \in\{0,1\}, w t\left(a_{1}, \ldots, a_{n-t}\right)=n-w\right\},
$$

is an anticode in $J(n, n-w)$ whose diameter is $w-t$ and its size is $\left(\begin{array}{l}n-t \\ w-t\end{array}\right)$.

Proof. Clearly, $\overline{\mathcal{A}}(n, w, t)$ is the complement of $\mathcal{A}(n, w, t)$ and hence by Lemma 4 , we have that $|\overline{\mathcal{A}}(n, w, t)|=\left(\begin{array}{c}n-t \\ w-t\end{array}\right)$. Moreover, for each two words $x, y \in \mathrm{J}(n, w), d(x, y)=d(\bar{x}, \bar{y})$ and hence the diameter of $\overline{\mathcal{A}}(n, w, t)$ equals to the diameter of $\mathcal{A}(n, w, t)$ which is $w-t$ by Lemma 4. Thus, the claim of the lemma follows directly from Lemma 4 . 
Definition 4. A Steiner system $S(t, w, n)$ is a pair $S=(\mathcal{N}, B)$, where $\mathcal{N}$ is an n-set and $B$ is a set of w-subsets (called blocks) from $\mathcal{N}$, where each $t$-subset of $\mathcal{N}$ is contained in exactly one block of $B$.

Subsets can be translated to words, and vice versa, via the following definition. The characteristic vector of a $w$-subset $S$ of an $n$-set $\mathcal{N}$ is a binary word of length $n$ and weight $w$ whose $i$-th coordinate is a one if and only if the $i$-th element of $\mathcal{N}$ is contained in $S$. In some cases, we are going to consider mixed language of words and subsets and the translation between the two should be understood from the context.

The following well-known theorem is readily verified.

Theorem 9. The number of blocks in a Steiner system $S(t, w, n)$ is

$$
\left(\begin{array}{l}
n \\
t
\end{array}\right) /\left(\begin{array}{l}
w \\
t
\end{array}\right)
$$

and its minimum Johnson distance is $w-t+1$.

Theorem 10. Any Steiner system $S(t, w, n)$ forms a $(w-t)$-diameter perfect code.

Proof. If $\mathcal{C}$ is the code constructed from a Steiner system $\mathrm{S}(t, w, n)$, then by Theorem 9 its Johnson distance is $w-t+1$, and

$$
|\mathcal{C}|=\frac{\left(\begin{array}{c}
n \\
t
\end{array}\right)}{\left(\begin{array}{c}
w \\
t
\end{array}\right)}=\frac{\left(\begin{array}{c}
n \\
w
\end{array}\right)}{\left(\begin{array}{c}
n-t \\
w-t
\end{array}\right)}
$$

On the other hand by the code-anticode bound $|\mathcal{C}| \cdot|\mathcal{A}| \leq\left(\begin{array}{l}n \\ w\end{array}\right)$, where $\mathcal{A}$ is an anticode in $\mathrm{J}(n, w)$ whose diameter is $w-t$, and therefore $|\mathcal{A}| \leq\left(\begin{array}{c}n-t \\ w-t\end{array}\right)$.

Since by Lemma 4 the set $\mathcal{A}(n, w, t)$ is an anticode in $\mathrm{J}(n, w)$ of size $\left(\begin{array}{l}n-t \\ w-t\end{array}\right)$ and whose diameter is $w-t$, the claim of the theorem follows.

Theorem 10 was proved in [1, but it should be noted (as it was not mentioned in [1]), that except for the family of Steiner systems, there is another family of diameter perfect codes in $\mathrm{J}(n, w)$.

Theorem 11. The complement of a Steiner system $S(t, w, n)$ forms a $(w-t)$-diameter perfect code.

Proof. For each two words $x, y \in \mathrm{J}(n, w), d(x, y)=d(\bar{x}, \bar{y})$ and hence the complement of a Steiner system $\mathrm{S}(t, w, n)$ has minimum Johnson distance $w-t+1$. By Lemma 5 , $\overline{\mathcal{A}}(n, w, t)$ and $\mathcal{A}(n, w, t)$ have the same diameter $w-t$ and the same size $\left(\begin{array}{c}n-t \\ w-t\end{array}\right)$. Moreover, the weight of the codewords in $\overline{\mathcal{A}}(n, w, t)$ is $n-w$ and since also $\left(\begin{array}{c}n \\ n-w\end{array}\right)=\left(\begin{array}{c}n \\ w\end{array}\right)$, this implies, as in the proof of Theorem 10, that the complement of a Steiner system $\mathrm{S}(t, w, n)$ forms a $(w-t)$-diameter perfect code in $\mathrm{J}(n, n-w)$.

Corollary 3. Any Steiner system $S(t, w, n)$ and any complement of a Steiner system $S(t, w, n)$ forms a $(w-t)$-diameter perfect code. 
There is a well-known conjecture [12] that there are no nontrivial perfect codes in $\mathrm{J}(n, w)$. Steiner systems are embedded in diameter perfect codes in $\mathrm{J}(n, w)$ [1] similar to Steiner systems embedded in perfect codes in $\mathrm{J}(n, w)$ as was proved in [15]. This makes it very tempting to have a similar conjecture for diameter perfect codes.

Conjecture 1. There are no nontrivial diameter perfect codes in $J(n, w)$, except for Steiner systems and their complements.

The following well-known results, to which we will refer later, can be easily verified.

Lemma 6. If there exists a Steiner system $S(t, w, n), t>1$, then there exists a Steiner system $S(t-1, w-1, n-1)$.

Corollary 4. A necessary condition that a Steiner system $S(t, w, n)$ exists is that all the numbers $\frac{\left(\begin{array}{c}n-i \\ t-i\end{array}\right)}{\left(\begin{array}{c}k-i \\ t-i\end{array}\right)}, 0 \leq i \leq t-1$, are integers.

Steiner system were investigated throughout the years and a short survey by Colbourn and Mathon can be found in [11, pp. 102-110]. It was proved in [23] and later in [21] that for each pair $(t, w)$, there exists an $n_{0}$ such that for each $n \geq n_{0}$ the necessary conditions of Corollary 4 are also sufficient. Unfortunately, the proof is nonconstructive and this $n_{0}$ is beyond our imagination.

\section{Non-Binary Diameter Perfect Constant-Weight Codes}

The main part of our paper is devoted to non-binary diameter perfect constant-weight codes. We distinguish between six families of such codes in $\mathrm{J}_{q}(n, w)$, where $q>2$.

[F1] Non-binary diameter perfect constant-weight codes for which $w=n$.

[F2] Diameter perfect constant-weight codes over an alphabet of size $2^{k}+1$ for which $w=n-1$

[F3] Non-binary diameter perfect constant-weight codes which are generalized Steiner systems.

[F4] Non-binary diameter perfect constant-weight codes for which $d=w$. These codes are called maximum distance separable constant-weight codes. Each such code has $\left(\begin{array}{l}n \\ w\end{array}\right)(q-1)$ codewords.

[F5] Non-binary diameter perfect constant-weight codes for which $d=w+1$. Such a code has $\left(\begin{array}{l}n \\ w\end{array}\right)$ codewords.

[F6] Non-binary diameter perfect constant-weight codes for which $d<w$. These codes are called multiple orthogonal arrays constant-weight codes. Each such code has $\left(\begin{array}{l}n \\ w\end{array}\right)(q-1)^{w-d+1}$ codewords.

Before we start our discussion on these six families of codes we have to prove the local inequality lemma for $\mathrm{J}_{q}(n, w)$ with the Hamming metric. For the proof of this lemma it is required to prove the following simple lemma. 
Lemma 7. For each $q \geq 2$ and any given pair $(t, n)$, where $1 \leq t \leq n$, there exists some $\lambda \geq 1$ for which there exists an $O A_{\lambda}(t, n, q)$.

Proof. Consider a matrix $\mathcal{M}$ whose rows are all the $q^{n}$ distinct words of length $n$ over $\mathbb{Z}_{q}$. Clearly, in each projection of $t$ coordinates from $\mathcal{M}$ each $t$-tuple is contained in $\frac{q^{n}}{q^{t}}=q^{n-t}$ distinct rows (codewords). Thus, the $q^{n} \times n$ matrix $\mathcal{M}$ forms an $\mathrm{OA}_{\lambda}(t, n, q)$, where $\lambda=q^{n-t}$.

Lemma 8. Let $\mathcal{C}_{\mathcal{D}}$ be a constant-weight code of length $n$ and weight $w$ over $\mathbb{Z}_{q}, q>2$, with distances between the codewords of $\mathcal{C}_{\mathcal{D}}$ taken from a subset $\mathcal{D}$. Let $\mathcal{A}$ be a subset of $J_{q}(n, w)$ and let $\mathcal{C}_{\mathcal{D}}^{\prime} \subseteq \mathcal{A}$ be the largest code in $\mathcal{A}$ with distances taken from $\mathcal{D}$. Then

$$
\frac{\left|\mathcal{C}_{\mathcal{D}}\right|}{\left(\begin{array}{l}
n \\
w
\end{array}\right)(q-1)^{w}} \leq \frac{\left|\mathcal{C}_{\mathcal{D}}^{\prime}\right|}{|\mathcal{A}|}
$$

Proof. Consider the set of pairs

$$
\mathcal{P}=\left\{(c, \pi): c \in \mathcal{C}_{\mathcal{D}}, \pi \in S_{n}, \operatorname{supp}(\pi(c))=\operatorname{supp}(a), \text { where } a \in \mathcal{A}\right\} \text {. }
$$

For a fixed $c \in \mathcal{C}_{\mathcal{D}}$ and a fixed $a \in \mathcal{A}$ there are exactly $w !(n-w)$ ! choices for $\pi$, for which $\operatorname{supp}(\pi(c))=\operatorname{supp}(a)$. Hence, the number of pairs in $\mathcal{P}$ equals to $\left|\mathcal{C}_{\mathcal{D}}\right| \cdot|\mathcal{A}| \cdot w ! \cdot(n-w)$ !.

For the word $v=\left(v_{1}, v_{2}, \ldots, v_{n}\right) \in \mathbb{Z}_{q-1}^{n}$, we form a subset $\mathcal{A}_{v}$ of $\mathrm{J}_{q}(n, w)$ as follows. Given a word $x=\left(x_{1}, x_{2}, \ldots, x_{n}\right)$ of $\mathcal{A}$, the word $a_{v}=\left(a_{1}, a_{2}, \ldots, a_{n}\right)$ is constructed in $\mathcal{A}_{v}$ as follows.

1. If $x_{i}=0$, then $a_{i}=x_{i}=0$.

2. If $x_{i} \neq 0$, then $a_{i}=x_{i}+v_{i}$ when $x_{i}+v_{i}<q$ and $a_{i}=x_{i}+v_{i}-(q-1)$ when $x_{i}+v_{i} \geq q$. In other words, if $j=v_{i}$, then $a_{i}$ takes the $j$-th nonzero value of $\mathbb{Z}_{q}$ after the value of $x_{i}$, where 1 follows $q-1$.

Using this definition, we have that $\operatorname{supp}\left(a_{v}\right)=\operatorname{supp}(x)$.

Clearly, $\mathcal{A}_{v}$ is obtained from $\mathcal{A}$ by permuting the nonzero elements in each one of the $w$ nonzero coordinates, of the words in $\mathcal{A}$, by some $w$ cyclic permutations (a permutation for each coordinate) on the $q-1$ nonzero symbols of $\mathbb{Z}_{q}$ (which can be different for each coordinate) and hence $\left|\mathcal{A}_{v}\right|=|\mathcal{A}|$. Moreover, $\mathcal{A}_{v}$ and $\mathcal{A}$ are isomorphic subsets of $\mathrm{J}_{q}(n, w)$.

Now, let $\mathcal{M}$ be any orthogonal array $\mathrm{OA}_{\lambda}(w, n, q-1)$, for some $\lambda \geq 1$, whose existence is implied by Lemma 7 . The number of rows of $M$ is $\lambda(q-1)^{w}$.

Consider now the set of triples

$$
\mathcal{T}=\left\{(c, \pi, v): c \in \mathcal{C}_{\mathcal{D}}, \pi \in S_{n}, v \in \mathcal{M}, \pi(c) \in \mathcal{A}_{v}\right\}
$$

Let $(c, \pi)$ be a pair in $\mathcal{P}$, i.e., $c \in \mathcal{C}_{\mathcal{D}}, \pi \in S_{n}$, and $\operatorname{supp}(\pi(c))=\operatorname{supp}(a)$ for some $a \in \mathcal{A}$. Let $X=\operatorname{supp}(a)$ and let $u=\left(u_{1}, u_{2}, \ldots, u_{n}\right)$ be a word in $\mathbb{Z}_{q-1}^{n}$ such that $a=\pi(c)_{u}$. It is easy to verify that for each word $v \in \mathbb{Z}_{q-1}^{n}$, for which the projection of the coordinates in $X$ on $u$ and the projection of the coordinates in $X$ on $v$ are equal, we have that $\pi(c)_{v}=a=\pi(c)_{u}$. Since $\mathcal{M}$ contains $\lambda$ rows for which these projections are equal, it follows that $|\mathcal{T}|=\lambda|\mathcal{P}|$. 
Note, that for each permutation $\pi \in S_{n}$ and two elements $x, y \in \mathrm{J}_{q}(n, w)$, we have that $d(\pi(x), \pi(y))=d(x, y)$. This implies that a fixed permutation $\pi$ with a fixed row $v \in \mathcal{M}$ can transfer the elements of $\mathcal{C}_{\mathcal{D}}$ into at most $\left|\mathcal{C}_{\mathcal{D}}^{\prime}\right|$ elements of $\mathcal{A}_{v}$. Therefore, the number of triples in $\mathcal{T}$ is at most $\lambda \cdot\left|\mathcal{C}_{\mathcal{D}}^{\prime}\right| \cdot n ! \cdot(q-1)^{w}$ which implies that

$$
\lambda \cdot\left|\mathcal{C}_{\mathcal{D}}\right| \cdot|\mathcal{A}| \cdot w ! \cdot(n-w) !=\lambda \cdot|\mathcal{P}|=|\mathcal{T}| \leq \lambda \cdot\left|\mathcal{C}_{\mathcal{D}}^{\prime}\right| \cdot n ! \cdot(q-1)^{w},
$$

and hence the claim of the lemma is proved.

The important consequence from Lemma 8 is that (11) and (2) are satisfied and hence we can consider diameter perfect codes in $\mathrm{J}_{q}(n, w)$.

\subsection{Diameter perfect constant-weight codes for which $w=n$}

The first family, $[\mathbf{F 1}]$, of non-binary diameter perfect constant-weight codes contains all perfect code in the Hamming scheme and all diameter perfect codes in the Hamming scheme. Since by Theorem 3 any perfect code in the Hamming scheme is also a diameter perfect code, it follows that we can consider only diameter perfect codes. In this family of codes we have that $w=n$ and the size of the non-binary alphabet is increased by one compared to the alphabet in the Hamming scheme. The relation between these families of codes is stated in the following theorem.

Theorem 12. There exists a D-diameter perfect code of length $n$ over an alphabet of size q-1 in the Hamming scheme, if and only if there exists a D-diameter perfect constantweight code of length $n$ and weight $w=n$ over an alphabet of size $q$.

Proof. Let $\mathcal{C}$ be a $D$-diameter perfect code of length $n$ over the alphabet $\{1,2, \ldots, q-1\}$ in the Hamming scheme. Let $\mathcal{A}$ be the related maximum size anticode with diameter $D$ for which $|\mathcal{C}| \cdot|\mathcal{A}|=(q-1)^{n}$. We define the same code $\mathcal{C}^{\prime} \triangleq \mathcal{C}$ and the same anticode $\mathcal{A}^{\prime} \triangleq \mathcal{A}$, over the extended alphabet $Q=\{0,1,2, \ldots, q-1\}$. We claim that $\mathcal{C}^{\prime}$ is a $D$-diameter perfect constant-weight code of length $n$, weight $w=n$, and minimum Hamming distance $D+1$, over $Q$. We also claim that $\mathcal{A}^{\prime}$ is a maximum size anticode of length $n$, weight $w=n$, and maximum Hamming diatance $D$, over $Q$. Clearly, the minimum Hamming distance of $\mathcal{C}^{\prime}$ is equal to the minimum Hamming distance of $\mathcal{C}$, i.e., $D+1$. Similarly, the maximum Hamming distance of $\mathcal{A}^{\prime}$ is equal to the maximum Hamming distance of $\mathcal{A}$, i.e., D. Moreover,

$$
\left|\mathcal{C}^{\prime}\right| \cdot\left|\mathcal{A}^{\prime}\right|=|\mathcal{C}| \cdot|\mathcal{A}|=(q-1)^{n}=\left|\mathrm{J}_{q}(n, n)\right|
$$

which completes the proof of our claims.

Let $\mathcal{C}$ be an $D$-diameter perfect constant-weight code of length $n$ and weight $w=n$ over the alphabet $Q=\{0,1,2, \ldots, q-1\}$. Using similar arguments, in reverse order, the same code defined over $Q^{*}$ is a $D$-diameter perfect code, of length $n$ over $Q^{*}$, in the Hamming scheme. Similarly, if $\mathcal{A}$ is a maximum size anticode, of length $n$ and weight $w=n$, with diameter $D$, over $Q$, then the same anticode defined over $Q^{*}$ is a maximum size anticode over $Q^{*}$. 
In other words, Theorem 12 implies that when $w=n$, in each word all the coordinates are nonzero. Hence, the words in $\mathrm{J}_{q}(n, n)$ are over an alphabet with only $q-1$ letters. It implies that any code in $\mathrm{J}_{q}(n, n)$ can be considered as a code in the Hamming scheme over an alphabet with $q-1$ letters. Therefore, any $D$-diameter perfect code of length $n$ over an alphabet with $q-1$ letters is also $D$-diameter perfect code in $\mathrm{J}_{q}(n, n)$. Similarly, each maximum size anticode of length $n$ and diameter $D$ over an alphabet with $q-1$ letters (with no zeroes) is also a maximum size anticode with diameter $D$ in $\mathrm{J}_{q}(n, n)$. Finally, all the diameter perfect codes in the Hamming scheme over an alphabet whose size is a prime power were characterized in [1].

Theorem 13. In the Hamming scheme whose alphabet size is a prime power there are no diameter perfect codes except for the Hamming codes, the extended Hamming codes, the Golay codes, the extended Golay codes, and the MDS codes.

When $q$ is not a prime power the only known diameter perfect codes are the orthogonal arrays with index unity.

\subsection{Codes with alphabet size $2^{k}+1$ for which $w=n-1$}

By Theorem 4 an $e$-perfect code in $\mathrm{J}_{q}(n, w)$ is also a $(2 e)$-diameter perfect code in $\mathrm{J}_{q}(n, w)$. All known nontrivial non-binary perfect constant-weight codes of length $n$ have weight $w=$ $n-1$ and they form an important class of the family [F2] of non-binary diameter perfect codes. There are a few known families of nontrivial non-binary perfect constant-weight codes. Ternary 1-perfect codes in $\mathrm{J}_{3}\left(2^{m}, 2^{m}-1\right), m \geq 2$, were constructed in [32, 34] and it was proved in [34] that there are no other ternary 1-perfect constant-weight codes. A large number of nonequivalent codes with these parameters were constructed by [24]. The construction in [34] was generalized in [19] and 1-perfect codes were constructed in $\mathrm{J}_{q}(q+1, q)$, where $q=2^{k}+1, k \geq 2$. It is not known whether there exist more nontrivial perfect constant-weight codes.

Ternary diameter perfect codes of length $n$ and weight $w=n-1$ were considered in [24]. For $d=4$, it was proved in [24] that there is only one set of parameters for which there exist a ternary 3 -diameter perfect constant-weight codes of length $n$ and weight $w=n-1$. A ternary code in this set has length 6 , weight 5, and 12 codewords. Such a code was constructed in [28]. For ternary 4-diameter perfect constant-weight codes, it was proved by [24] that for every length $n=2^{m}, m$ odd, there exists such a code with weight $w=n-1$. When $m$ is even only a code of length $n=64$ constructed in [5] is known [25] and it was proved in [25] that such a code does not exist for length $n=16$.

\subsection{Generalized Steiner systems}

We already saw that a Steiner system $\mathrm{S}(t, w, n)$ is a binary $(w-t)$-diameter perfect constantweight code. For non-binary alphabet, there is an analog definition of generalized Steiner system which was introduced in [16].

Definition 5. A generalized Steiner system $G S(t, w, n, q)$ is a constant-weight code $\mathcal{C}$, over $\mathbb{Z}_{q}$, whose length is $n$, weight $w$, for each codeword, such that: 
1. The minimum Hamming distance of $\mathcal{C}$ is $2(w-t)+1$.

2. Each word $x$ of length $n$ and weight $t$ over $\mathbb{Z}_{q}$ is covered by exactly one codeword $c \in \mathcal{C}$, i.e., $d(x, c)=w-t$.

Similarly to Theorem 9 we have the following theorem.

Theorem 14. The number of codewords in a generalized Steiner system $G S(t, w, n, q)$ is

$$
\frac{\left(\begin{array}{c}
n \\
t
\end{array}\right)}{\left(\begin{array}{c}
w \\
t
\end{array}\right)}(q-1)^{t}
$$

and its minimum Hamming distance is $2(w-t)+1$.

Similarly to Lemma 6 one can verify the following lemma.

Lemma 9. If there exists a $G S(t, w, n, q), t>1$, then there exists a $G S(t-1, w-1, n-1, q)$.

Let $\mathcal{A}^{\mathrm{s}}(n, w, t)$ be the anticode defined by

$$
\mathcal{A}^{\mathrm{s}}(n, w, t) \triangleq\left\{(\overbrace{1 \cdots \cdots 1}^{t \text { times }}, a_{1}, \ldots, a_{n-t}): a_{i} \in \mathbb{Z}_{q}, \operatorname{wt}\left(a_{1} \cdots a_{n-t}\right)=w-t\right\} .
$$

Note, that when $q=2$ we have that $\mathcal{A}^{\mathrm{s}}(n, w, t)$ is identical to $\mathcal{A}(n, w, t)$.

The following lemma can be readily verified.

Lemma 10. The anticode $\mathcal{A}^{s}(n, w, t)$ has codewords of length $n$ and weight $w$, with maximum distance $2(w-t)$, where $n \leq 2 w-t$, over $\mathbb{Z}_{q}$. The anticode $\mathcal{A}^{s}(n, w, t)$ has $\left(\begin{array}{c}n-t \\ w-t\end{array}\right)(q-1)^{w-t}$ codewords.

Lemma 11. If there exists a generalized Steiner system $S(t, w, n, q)$, then the anticode $\mathcal{A}^{s}(n, w, t)$ is a maximum size anticode of length $n$ and weight $w$, with maximum distance $2(w-t)$, where $n \leq 2 w-t$, over $\mathbb{Z}_{q}$.

Proof. Let $\mathcal{C}$ be a generalized Steiner system $\operatorname{GS}(t, w, n, q)$ and let $\mathcal{A}$ be the anticode $\mathcal{A}^{\mathrm{s}}(n, w, t)$. By the definition of a generalized Steiner system and by Lemma 14, $\mathcal{C}$ has minimum Hamming distance $2(w-t)+1$ and its size is $\frac{\left(\begin{array}{c}n \\ t\end{array}\right)}{\left(\begin{array}{c}w \\ t\end{array}\right)}(q-1)^{t}$. By Lemma 10 the anticode $\mathcal{A}^{\mathrm{s}}(n, w, t)$ has maximum distance $2(w-t)$ and its size is $\left(\begin{array}{c}n-t \\ w-t\end{array}\right)(q-1)^{w-t}$. Since

$$
|\mathcal{C}| \cdot|\mathcal{A}|=\frac{\left(\begin{array}{c}
n \\
t
\end{array}\right)}{\left(\begin{array}{c}
w \\
t
\end{array}\right)}(q-1)^{t} \cdot\left(\begin{array}{c}
n-t \\
w-t
\end{array}\right)(q-1)^{w-t}=\left(\begin{array}{c}
n \\
w
\end{array}\right)(q-1)^{w}=\left|\mathrm{J}_{q}(n, w)\right|
$$

it follows by the code-anticode bound that $\mathcal{A}^{\mathrm{s}}(n, w, t)$ is a maximum size anticode of length $n$ and weight $w$, over $\mathbb{Z}_{q}$, whose maximum distance $2(w-t)$.

Corollary 5. A generalized Steiner system $G S(t, w, n, q)$ is a $2(w-t)$-diameter perfect code.

Generalized Stiener systems were defined in [16] and further considered in many papers, e.g. [7, 10, 29, 35], but there is still lot ground for further research in this area. 


\subsection{Maximum distance separable constant weight codes}

Definition 6. An $(n, w, q)$ maximum distance separable constant-weight code (MDS$C W$ code in short) is a constant-weight code $\mathcal{C}$, over $\mathbb{Z}_{q}$, whose length is $n$, weight $w$, for each codeword, such that:

1. The minimum Hamming distance of $\mathcal{C}$ is $w$.

2. Each subset of $w$ coordinates is the support of exactly $q-1$ codewords.

The name MDS-CW codes is a consequence from the observation that the minimum weight codewords in an MDS codes form such an MDS-CW code [27, pp. 319-321].

Let $\mathcal{A}^{\mathrm{m}}(n, w, \delta), 1 \leq \delta \leq w$, be the anticode defined as follows

$$
\mathcal{A}^{\mathrm{m}}(n, w, \delta) \triangleq\left\{(a_{1}, a_{2}, \ldots, a_{\delta}, \overbrace{1 \cdots \cdots 1}^{w-\delta \text { times }}, \overbrace{0 \cdots \cdots 0}^{n-w}): a_{i} \in \mathbb{Z}_{q} \backslash\{0\}\right\} .
$$

The following lemma can be readily verified.

Lemma 12. The anticode $\mathcal{A}^{m}(n, w, \delta)$ has codewords of length $n$, weight $w$, with maximum distance $\delta$, and the number of codewords in $\mathcal{A}^{m}(n, w, \delta)$ is $(q-1)^{\delta}$.

Lemma 13. If there exists an $(n, w, q) M D S-C W$ code, then the anticode $\mathcal{A}^{m}(n, w, w-1)$ is a maximum size anticode of length $n$, weight $w$, with maximum distance $w-1$, over $\mathbb{Z}_{q}$.

Proof. Let $\mathcal{C}$ be an $(n, w, q)$ MDS-CW code and $\mathcal{A}$ be the anticode $\mathcal{A}^{\mathrm{m}}(n, w, w-1)$. By the definition, an $(n, w, q)$ MDS-CW code, has minimum distance $w$ and size $\left(\begin{array}{c}n \\ w\end{array}\right)(q-1)$. By Lemma 12, the anticode $\mathcal{A}^{\mathrm{m}}(n, w, w-1)$ has maximum distance $w-1$ and size $(q-1)^{w-1}$. Since

$$
|\mathcal{C}| \cdot|\mathcal{A}|=\left(\begin{array}{l}
n \\
w
\end{array}\right)(q-1) \cdot(q-1)^{w-1}=\left(\begin{array}{l}
n \\
w
\end{array}\right)(q-1)^{w}=\left|\mathrm{J}_{q}(n, w)\right|
$$

it follows by the code-anticode bound that the anticode $\mathcal{A}^{\mathrm{m}}(n, w, w-1)$ is a maximum size anticode of length $n$, weight $w$, with maximum distance $w-1$, over $\mathbb{Z}_{q}$.

Corollary 6. An $(n, w, q) M D S-C W$ code is a $(w-1)$-diameter perfect code.

Given a pair $(w, n)$ it is very simple to verify from Theorem 8 that there exists a prime power $q$ for which there exists an $(n, w, q)$ MDS-CW code. But, this can be further improved as It was proved in [16] that there exists a $q_{0}=Q M D S(n, w)$ such that for each $q \geq q_{0}$ there exists an $(n, w, q)$ MDS-CW code. Finally, we will present a theorem related to a very simple union construction [16] which can be used to prove an upper bound on this $Q M D S(n, w)$.

Theorem 15. If there exists an $\left(n, w, q_{1}\right) M D S-C W$ code and an $\left(n, w, q_{2}\right) M D S-C W$ code, then there exists an $\left(n, w, q_{1}+q_{2}-1\right) M D S-C W$ code.

There are more constructions and bounds on the parameters of MDS-CW codes which were presented in [16]. Some of these bounds and constructions can be modified and generalized to the sixth family, [F6], which will be discussed in Section 4.6. 


\subsection{Codes for which $d=w+1$}

When $d=w+1$ we are looking for an $(n, w+1, w)_{q}$ code, i.e., a constant-weight code of length $n$, weight $w$, and minimum Hamming distance $w+1$, over $\mathbb{Z}_{q}$. In such a code, each subset of $w$ coordinates will support exactly one codeword, i.e., the number of codewords is $\left(\begin{array}{l}n \\ w\end{array}\right)$. It is rather easy to verify that such a code is a $w$-diameter perfect constant-weight code and it exists for any given $n$ and $w$ as it is proved in the following theorem.

Theorem 16. If $n$ and $w$ are integers such that $1 \leq w \leq n-1$, then there exists a $q_{0}(w, n)$ such that for each $q \geq q_{0}(w, n)$ there exist an $(n, w+1, w)_{q} w$-diameter perfect code $\mathcal{C}$.

Proof. First, note that since the minimum distance of an $(n, w+1, w)_{q}$ code $\mathcal{C}$ is $w+1$, it follows that each subset of $w$ coordinates can be a support for at most one codeword. If each such subset of $w$ coordinates supports exactly one codeword, then the total number of codewords in $\mathcal{C}$ will be $\left(\begin{array}{l}n \\ w\end{array}\right)$. Assume further that in $\mathcal{C}$ for each coordinate all the nonzero elements in the codewords of $\mathcal{C}$ have distinct symbols. It implies that in each coordinate there are $\left(\begin{array}{l}n \\ w\end{array}\right) \frac{w}{n}=\left(\begin{array}{l}n-1 \\ w-1\end{array}\right)$ nonzero symbols. Let $q^{\prime} \triangleq 1+\left(\begin{array}{c}n-1 \\ w-1\end{array}\right)$ and let $Q$ be an alphabet with $q=q^{\prime}+\epsilon$ symbols, where $\epsilon \geq 0$. Assign now for each coordinate a different nonzero symbols from the $q^{\prime}+\epsilon-1$ nonzero symbols of $Q^{*}$ to each codeword that has a nonzero symbol in this coordinate. Clearly, $\mathcal{C}$ in an $(n, w+1, w)_{q}$ code with $\left(\begin{array}{l}n \\ w\end{array}\right)$ codewords.

Let $\mathcal{A}$ be the anticode $\mathcal{A}^{\mathrm{m}}(n, w, w)$ over $Q$. By Lemma 12, the anticode $\mathcal{A}$, has diameter $w$ and $(q-1)^{w}$ codewords. Clearly,

$$
|\mathcal{C}| \cdot|\mathcal{A}|=\left(\begin{array}{c}
n \\
w
\end{array}\right)(q-1)^{w}=\left|J_{q}(n, w)\right|
$$

and hence by the code-anticode bound, $\mathcal{C}$ is an $(n, w+1, w)_{q}$ w-diameter perfect constantweight code over the alphabet $Q$ of size $q$.

Corollary 7. If there exists an $(n, w+1, w)_{q}$ code with $\left(\begin{array}{l}n \\ w\end{array}\right)$ codewords, then $\mathcal{A}^{m}(n, w, w)$ is a maximum size anticode of length $n$, weight $w$, and diameter $w$, over and alphabet with $q$ symbols.

The proof of Theorem 16 implies that an $(n, w+1, w)_{q} w$-diameter constant-weight perfect code $\mathcal{C}$ has $\left(\begin{array}{l}n \\ w\end{array}\right)$ codewords, where each $w$-subset of $w$ coordinates of $\mathcal{C}$ is the support of exactly one codeword of $\mathcal{C}$. In view of Theorem [16 our goal now is to find $q_{0}(w, n)$ which is the smallest size alphabet $q$ for such an $(n, w+1, w)_{q}$ code exists.

Corollary 8. For each alphabet $Q$ of size $q$, where $q \geq 1+\left(\begin{array}{c}n-1 \\ w-1\end{array}\right)$, there exists an $(n, w+1, w)_{q}$ code, i.e., $q_{0}(w, n) \leq 1+\left(\begin{array}{c}n-1 \\ w-1\end{array}\right)$.

Lemma 14. For each $w \geq 1$ there exists an $(w+1, w+1, w)_{w+1}$ code which is a $w$-diameter perfect code.

Proof. Follows immediately from the fact that if there is a codeword on each subset of $w$ coordinates, then there are exactly $w$ codewords with nonzero symbols on each coordinates.

Corollary 9. If $w \geq 1$, then $q_{0}(w, w+1)=w+1$. 
Theorem [16 implies the existence of an $(n, w+1, w)_{q}$ code for each $q \geq q_{0}(w, n)$, but the upper bound $1+\left(\begin{array}{c}n-1 \\ w-1\end{array}\right)$ on $q_{0}(w, n)$, inferred in Corollary 8 , is quite large. Can we find a better upper bound on $q_{0}(w, n)$ ? The answer is definitely positive and for this purpose we have the following results.

Lemma 15. If $n>w+1$, then $q_{0}(w, n) \geq q_{0}(w, n-1)$.

Proof. Assume that $\mathcal{C}$ is an $(n, w+1, w)_{q} w$-diameter constant-weight perfect code and let $S$ be any subset of $n-1$ coordinates. By definition, the set codewords whose supports are subsets of $S$ form an $(n-1, w+1, w)_{q} w$-diameter constant-weight perfect code. Thus, the claim of the lemma follows.

Corollary 10. If $n>w+1$ then $q_{0}(w, n) \geq w+1$.

Lemma 16. If $n>w+1$, then $q_{0}(w, n) \geq n-w+2$.

Proof. Let $\mathcal{C}$ be an $(n, w+1, w)_{q} w$-diameter perfect code $\mathcal{C}$. Consider the sub-code $\mathcal{C}^{\prime}$ of codewords from $\mathcal{C}$ for which there is no zero in the first $w-1$ coordinates. Since, each one of the other $n-w+1$ coordinates must have a nonzero symbol with exactly one of these codewords, it follows that the sub-code $\mathcal{C}^{\prime}$ contains $n-w+1$ codewords. Since the distance of $\mathcal{C}^{\prime}$ is $w+1$, each pair of codewords of $\mathcal{C}^{\prime}$ have only two distinct coordinates in their supports, and each pair of codewords of $\mathcal{C}^{\prime}$ have $w-1$ joint coordinates with nonzero symbols, it follows that in each given coordinate of the first $w-1$ coordinates the codewords of $\mathcal{C}^{\prime}$ have distinct nonzero symbols. Since $\left|\mathcal{C}^{\prime}\right|=n-w+1$, it follows that $\mathcal{C}$ has at least $n-w+1$ nonzero symbols and hence $q \geq n-w+2$.

Corollary 11. If $n>2$, then $q_{0}(2, n)=n$.

Proof. By Lemma 16 we have that $q_{0}(2, n) \geq n$ and by Corollary 8 we have that $q_{0}(2, n) \leq n$.

Thus, $q_{0}(2, n)=n$.

The proof of the next theorem requires two more concepts, a one-factorization and a near-one-factorization. A one-factorization of the complete graph $K_{n}, n$ even, is a partition of the edges of $K_{n}$ into perfect matchings. In other words, the set

$$
\mathcal{F}=\left\{\mathcal{F}_{1}, \mathcal{F}_{2}, \ldots, \mathcal{F}_{n-1}\right\}
$$

is a one-factorization of $K_{n}$ if each $\mathcal{F}_{i}, 1 \leq i \leq n-1$, is a perfect matching (called a one-factor), and the $\mathcal{F}_{i}$ 's are pairwise disjoint.

If $n$ is odd, then there is no perfect matching in $K_{n}$ and we define a near-one-factorization

$$
\mathcal{F}=\left\{\mathcal{F}_{1}, \mathcal{F}_{2}, \ldots, \mathcal{F}_{n}\right\}
$$

to be a partition of the edges in $K_{n}$ into sets of $\frac{n-1}{2}$ pairwise disjoint edges, where each $\mathcal{F}_{i}$ has one isolated vertex. Each $\mathcal{F}_{i}$ is called a near-one-factor.

Theorem 17. If $n$ is odd, then $q_{0}(3, n)=n-1$, and if $n$ is even, then $q_{0}(3, n)=n$. 
Proof. By Lemma 16 we have that $q_{0}(3, n) \geq n-1$ and this bound is applied when $n$ is odd.

Assume now that $n$ is even and let $\mathcal{C}$ be a related code. Let $\mathcal{C}_{1}$ be the set of codewords in $\mathcal{C}$ with a nonzero symbol in the first coordinate. By the definition of this family of codes, it follows that $\left|\mathcal{C}_{1}\right|=\left(\begin{array}{c}n-1 \\ 2\end{array}\right)=\frac{(n-1)(n-2)}{2}$. Since the minimum distance of $\mathcal{C}_{1}$ is four and $n$ is even, it follows that the number of codewords in $\mathcal{C}_{1}$ with a given nonzero symbol $\sigma$ in the first coordinate is at most $\frac{n-2}{2}$. Since $\left|\mathcal{C}_{1}\right|=\frac{(n-1)(n-2)}{2}$, it follows that there are at least $n-1$ nonzero symbols in the first coordinate. Therefore, $q_{0}(3, n) \geq n$ if $n$ is even.

Regarding the upper bound on $q_{0}(3, n)$ we distinguish again between two cases, depending on whether $n$ is odd or $n$ is even.

Case 1. $n$ is odd.

Let $\mathcal{N}$ be the set of $n$ coordinates and let $Q \triangleq\left\{0, \sigma_{1}, \ldots, \sigma_{n-2}\right\}$ be an alphabet of size $n-1$. Let $\mathcal{C}$ be a code of length $n$ and weight 3 with $\left(\begin{array}{l}n \\ 3\end{array}\right)$ codewords, a codeword for each support of size 3. Consider the $i$-th coordinate, $i \in \mathcal{N}$ and let $\mathcal{F}=\left\{\mathcal{F}_{1}, \mathcal{F}_{2}, \ldots, \mathcal{F}_{n-2}\right\}$ be a one-factorization on the $n-1$ points of $\mathcal{N} \backslash\{i\}$. Given a triple $\{i, j, k\}$, where $\{j, k\} \in \mathcal{F}_{r}$, we assign $\sigma_{r}$ to the symbol in coordinate $i$ of the codeword $\{i, j, k\}$, where the nonzero symbols are in coordinates $i, j, k$. It is readily verified that we have constructed a code of length $n$ and weight 3, over an alphabet $Q$ of size $n-1$. Clearly, if two codewords share at most one coordinate, then their Hamming distance is at least 4 . Now, assume that two codewords $c_{1}$ and $c_{2}$ share nonzero symbols in two coordinates $i$ and $j$. If the symbols in the $i$-th coordinate of $c_{1}$ and $c_{2}$ are distinct and the symbols in the $j$-th coordinate of $c_{1}$ and $c_{2}$ are distinct, then clearly $d\left(c_{1}, c_{2}\right)=4$. Now, assume for the contrary that in one coordinate, say $i, c_{1}$ and $c_{2}$ have the same symbol. By the construction, we have that the two other pairs of nonzero coordinates in $c_{1}$ and $c_{2}$ must be disjoint (they belong to the same one-factor), a contradiction. Therefore, the minimum distance of $\mathcal{C}$ is 4 and hence $q_{0}(3, n) \leq n-1$.

Case 2. $n$ is even.

Let $\mathcal{N}$ be the set of $n$ coordinates and let $Q \triangleq\left\{0, \sigma_{1}, \ldots, \sigma_{n-1}\right\}$ be an alphabet of size $n$. Let $\mathcal{C}$ be a code of length $n$ and weight 3 with $\left(\begin{array}{l}n \\ 3\end{array}\right)$ codewords, a codeword for each support of size 3. Consider the $i$-th coordinate, $i \in \mathcal{N}$ and let $\mathcal{F}=\left\{\mathcal{F}_{1}, \mathcal{F}_{2}, \ldots, \mathcal{F}_{n-1}\right\}$ be a near-onefactorization on the $n-1$ points of $\mathcal{N} \backslash\{i\}$. Given a triple $\{i, j, k\}$, where $\{j, k\} \in \mathcal{F}_{r}$, we assign $\sigma_{r}$ to the symbol in coordinate $i$ of the codeword $\{i, j, k\}$. It is readily verified that we have constructed a code of length $n$ and weight 3 , over an alphabet $Q$ of size $n$. As in Case 1 the minimum distance of $\mathcal{C}$ is 4 and therefore, $q_{0}(3, n) \leq n$.

Thus, these two cases complete the proof of the theorem.

Other codes with the same parameters as the ones constructed in Theorem 17 were also presented in [8, 9], by using different techniques from combinatorial designs. Similarly to the technique used in the proof of Theorem 17 one can construct $(n, w+1, w)_{q} w$-diameter perfect codes, for relatively small $q$, when $w$ is small using techniques coming from combinatorial designs. The same is true for $(n, w+1, w)_{q} w$-diameter perfect codes, when $n$ is not much larger than $w$. Such constructions are left for future research. Moreover, the technique used in Theorem 17 to obtain the upper bound on $q_{0}(3, n)$ can be used to obtain better upper bounds on $q_{0}(w+1, n+1)$ than the trivial one, i.e., $q_{0}(w+1, n+1) \leq 1+\left(\begin{array}{c}n \\ w\end{array}\right)$. The idea is to partition the set of all binary words of length $n$ and weight $w$ into pairwise disjoint constant-weight codes of length $n$, weight $w$, and minimum Hamming distance $w+2$. Let $\chi(n, w)$ be the minimum number of codes in such a partition. With an identical proof 
as the one in Theorem 17 we can prove that $q_{0}(w+1, n+1) \leq \chi(n, w)+1$. Note, that the minimum distance of a constant-weight code is always even and hence the proof will be more effective for even $w$, i.e., for bounds on $q_{0}(w+1, n+1)$ when $w+1$ is odd. This kind of a partition problem was discussed in [4]. As a simple example we can use prove the following bound implied by a result proved in [22].

Theorem 18. If $w$ is even and $p$ is the smallest prime power for which $p \geq n$, then

$$
q_{0}(w+1, n+1) \leq 1+p^{w / 2} .
$$

We leave further improvements in this direction for future research.

\subsection{Multiple orthogonal arrays constant weight codes}

In the last family of diameter perfect constant-weight codes we have similarly as in families $[\mathbf{F 4}]$ and $[\mathbf{F 5}]$ that each projection of any $w$ coordinates supports some specified number of codeword codewords. The distinction from families $[\mathbf{F 4}]$ and $[\mathbf{F 5}]$ is that the minimum distance of the code in this family, $[\mathbf{F 6}]$, is strictly smaller than the weight of the codewords. More precisely we have the following definition.

Definition 7. An $(n, d, w)_{q}$ multiple orthogonal arrays constant weight (MOA-CW in short) code is a code of length $n$, constant weight $w$, minimum distance $d<w$, where each subset of $w$ coordinates is the support of exactly $(q-1)^{w-d+1}$ codewords, i.e., these codewords form an $O A(w-d+1, w, q-1)$.

One might asks why this family does not include the MDS-CW codes, where $d=w$. The two families of codes, $[\mathbf{F 4}]$ and $[\mathbf{F 6}]$ share some properties such as similar expression of their size (which is also shared by the family $[\mathbf{F 5}]$ ), they are both related to orthogonal arrays (MDS codes) in a way that codewords with no zeroes in the same $w$ coordinates form an orthogonal array. The main reason for the distinction between the two families is that an MDS-CW code either forms the codewords of minimum weight in an orthogonal array or has the same parameters as it would have had, if such an orthogonal array have been possible. There is no similar property for an MOA-CW code. The codewords of an MOA-CW code are not related to codewords of some weights in MDS codes or orthogonal arrays. Another important distinction is in the simple union construction of Theorem 15 which cannot be applied to MOA-CW codes. The similarity of the two families will be also demonstrated in one construction of such codes which is a joint construction for both families of codes. Similarly, some bounds on the tradeoff between the parameters of these codes are joint bounds for the two families of codes.

Theorem 19. An $(n, d, w)_{q} M O A-C W$ code is a $(d-1)$-diameter perfect constant-weight code.

Proof. By definition, the size of an $(n, d, w)_{q}$ MOA-CW code $\mathcal{C}$ is $\left(\begin{array}{l}n \\ w\end{array}\right)(q-1)^{w-d+1}$ and by Lemma 12 the related anticode $\mathcal{A}$ with diameter $d-1$ in $\mathrm{J}_{q}(n, w), \mathcal{A}^{m}(n, w, d-1)$, has size $(q-1)^{d-1}$. Therefore we have that,

$$
|\mathcal{C}| \cdot|\mathcal{A}|=\left(\begin{array}{l}
n \\
w
\end{array}\right)(q-1)^{w-d+1}(q-1)^{d-1}=\left(\begin{array}{c}
n \\
w
\end{array}\right)(q-1)^{w}=\left|J_{q}(n, w)\right|
$$


which by the code-anticode bound implies that $\mathcal{C}$ is a $(d-1)$-diameter perfect constant-weight code.

Corollary 12. If there exists an $(n, d, w)_{q} M O A-C W$ code, then $\mathcal{A}^{m}(n, w, d-1)$ is a maximum size anticode of length $n$, weight $w$, and diameter $d-1$, over an alphabet with $q$ symbols.

Next, we present a construction for MOA-CW codes which can also serve as a construction for MDS-CW codes. The construction is a generalization and a modification of a construction presented in [16]. Let $\mathcal{M}$ be an $\mathrm{OA}(t, n, q)$ over $Q$, where $Q=\{1,2, \ldots, q\}$. Assume further that the symbols in the last coordinate of the first $q^{t-1}$ codewords of $\mathcal{M}$ are ones, the symbols in the last coordinate of the next $q^{t-1}$ codewords of $\mathcal{M}$ are twos, and so on, where the symbols in the last coordinate of the last $q^{t-1}$ codewords of $\mathcal{M}$ are $q$ 's. Assume further that $q \geq\left(\begin{array}{c}n-1 \\ \ell\end{array}\right)$ for a given $\ell, 1 \leq \ell \leq n-1$. Let $S_{1}, S_{2}, \ldots, S_{r}$, where $r=\left(\begin{array}{c}n-1 \\ \ell\end{array}\right)$, be a sequence containing all the $\ell$-subsets of $\{1,2, \ldots, n-1\}$. Let $\mathcal{M}^{\prime}$ be the $\left(\left(\begin{array}{c}n-1 \\ \ell\end{array}\right) q^{t-1}\right) \times(n-1)$ array constructed from $\mathcal{M}$ as follows.

1. If $S_{1}=\left\{i_{1}, i_{2}, \ldots, i_{\ell}\right\}$, then replace all the symbols in the first $q^{t-1}$ rows of column $i_{j}$ in $\mathcal{M}$, for each $1 \leq j \leq \ell$, with zeroes.

2. If $S_{2}=\left\{i_{1}, i_{2}, \ldots, i_{\ell}\right\}$, then replace all the symbols in the next $q^{t-1}$ rows of column $i_{j}$ in $\mathcal{M}$, for each $1 \leq j \leq \ell$, with zeroes.

3. Continue the same process with $S_{3}, S_{4}$, and so on until $S_{r}$.

4. Remove the last column of $\mathcal{M}$.

5. Remove the last $q^{t}-\left(\left(\begin{array}{c}n-1 \\ \ell\end{array}\right) q^{t-1}\right)$ rows of $\mathcal{M}$.

Theorem 20. The rows of the array $\mathcal{M}^{\prime}$ form an $(n-1, n-t-\ell+1, n-\ell-1)_{q+1}$ code $\mathcal{C}$ that is an $(n-t-\ell)$-diameter perfect constant-weight code over $Q \cup\{0\}$.

Proof. Exactly one column was deleted from $\mathcal{M}$ to obtain $\mathcal{M}^{\prime}$ and hence the length of the code $\mathcal{M}^{\prime}$ is $n-1$. In each codeword of length $n-1$ exactly $\ell$ zeroes were inserted instead of nonzero symbols and hence the weight of each codeword is $n-1-\ell$. Since $\mathcal{M}$ is an $\mathrm{OA}(t, n, q)$, it follows that $|\mathcal{M}|=q^{t}$, and since $q \geq\left(\begin{array}{c}n-1 \\ \ell\end{array}\right)$, it follows that $q^{t} \geq\left(\begin{array}{c}n-1 \\ \ell\end{array}\right) q^{t-1}$ and hence $\mathcal{M}$ has at least $\left(\begin{array}{c}n-1 \\ \ell\end{array}\right) q^{t-1}$ rows as required by the construction. Furthermore, note that the minimum distance of the code defined by $\mathcal{M}$ is $n-t+1$. Let $c_{1}$ and $c_{2}$ be two codewords in $\mathcal{M}^{\prime}$. If the zeroes in $c_{1}$ and $c_{2}$ are on the same $\ell$ coordinates, then $c_{1}$ and $c_{2}$ were derived from two rows $c_{1}^{\prime} \alpha$ and $c_{2}^{\prime} \alpha$ of $\mathcal{M}$, where $\alpha \in\{1,2, \ldots, q\}$, and $d\left(c_{1}^{\prime} \alpha, c_{2}^{\prime} \alpha\right) \geq n-t+1$. Since the same $\ell$ coordinates were changed in $c_{1}^{\prime}$ and $c_{2}^{\prime}$ to obtain $c_{1}$ and $c_{2}$, respectively, it follows that $d\left(c_{1}, c_{2}\right) \geq n-t+1-\ell$. If the zeroes in $c_{1}$ and $c_{2}$ are not on the same coordinates, then $c_{1}$ and $c_{2}$ were derived from two rows $c_{1}^{\prime} \alpha$ and $c_{2}^{\prime} \beta$, where $\alpha, \beta \in\{1,2, \ldots, q\}, \alpha \neq \beta$, and $d\left(c_{1}^{\prime} \alpha, c_{2}^{\prime} \beta\right) \geq n-t+1$, which implies that $d\left(c_{1}^{\prime}, c_{2}^{\prime}\right) \geq n-t$. The number of coordinates in which both $c_{1}$ and $c_{2}$ have zeroes is at most $\ell-1$ and hence $d\left(c_{1}, c_{2}\right) \geq d\left(c_{1}^{\prime}, c_{2}^{\prime}\right)-(\ell-1) \geq n-t-(\ell-1)=n-t-\ell+1$. Thus, $d(\mathcal{C}) \geq n-t-\ell+1$.

As an immediate consequence from the construction, the number of rows in the array $\mathcal{M}^{\prime}$ is $\left(\begin{array}{c}n-1 \\ \ell\end{array}\right) q^{t-1}$ and its alphabet $\{0,1,2, \ldots, q\}$ is of size $q+1$. Let $\mathcal{A}$ be a related anticode of 
length $n-1$ and diameter $n-t-\ell$. By Lemma 12 there exists such a constant-weight anticode $\mathcal{A}^{\mathrm{m}}(n-1, n-1-\ell, n-t-\ell)$, over $\mathbb{Z}_{q+1}$, whose size is $q^{n-t-\ell}$. Therefore,

$$
|\mathcal{C}| \cdot|\mathcal{A}|=\left(\begin{array}{c}
n-1 \\
\ell
\end{array}\right) q^{t-1} \cdot q^{n-t-\ell}=\left(\begin{array}{c}
n-1 \\
n-1-\ell
\end{array}\right) q^{n-\ell-1}=\left|\mathrm{J}_{q+1}(n-1, n-\ell-1)\right|
$$

which implies by the code-anticode bound that $\mathcal{M}^{\prime}$ is an $(n-t-\ell)$-diameter perfect code.

Corollary 13. When $t=2$ the code $\mathcal{M}^{\prime}$ is an $(n-1, n-\ell-1, q+1)$ MDS-CW code.

Corollary 14. When $t>2$ the code $\mathcal{M}^{\prime}$ is an $(n-1, n-t-\ell-1, n-\ell-1)_{q+1} M O A-C W$ code.

\section{Theorem 21.}

1. If there exists an $(n, d, w)_{q} M O A-C W$ code, then there exists an $(n-1, d, w)_{q} M O A-C W$ code.

2. If there exists an $(n, d, w)_{q} M O A-C W$ code, then there exists an $(n-1, d-1, w-1)_{q}$ $M O A-C W$ code.

Proof. Let $\mathcal{C}$ be $(n, d, w)_{q}$ MOA-CW code and define the following two code

$$
\mathcal{C}_{1} \triangleq\left\{\left(x_{2}, x_{3}, \ldots, x_{n}\right):\left(0, x_{2}, x_{3}, \ldots, x_{n}\right) \in \mathcal{C}\right\}
$$

and

$$
\mathcal{C}_{2} \triangleq\left\{\left(x_{2}, x_{3}, \ldots, x_{n}\right):\left(x_{1}, x_{2}, x_{3}, \ldots, x_{n}\right) \in \mathcal{C}, x_{1} \neq 0\right\} .
$$

One can easily verify that $\mathcal{C}_{1}$ is an $(n-1, d, w)_{q}$ MOA-CW code and $\mathcal{C}_{2}$ is an $(n-1, d-1, w-1)_{q}$ MOA-CW code.

After constructing $(d-1)$-diameter constant-weight perfect codes for $d<w$, where each $w$ coordinates are the support of exactly $(q-1)^{w-d+1}$ codewords we would like to have some lower bounds on the alphabet size of such codes and upper bounds on their length and their weights. Since each $w$ coordinates are the support of exactly $(q-1)^{w-d+1}$ codewords, it follows that the projection on each $w$ coordinates on these codewords forms an orthogonal array $\mathrm{OA}(w-d+1, w, q-1)$ and the related bounds on orthogonal arrays in Theorems 5, 6, and 7, can be applied. This implies the following theorem which present a tradeoff between the alphabet size and the minimum distance of the code.

\section{Theorem 22.}

1. If there exists an $(n, w-1, w)_{q} M O A-C W$ code, then $w \leq q$.

2. If there exists an $(n, w-\delta, w)_{q} M O A-C W$ code, where $2 \leq \delta \leq w-1$ and $q$ is even, then $w \leq q+\delta$.

3. If there exists an $(n, w-\delta, w)_{q} M O A-C W$ code, where $2 \leq \delta \leq w-1$ and $q$ is odd, then $w \leq q+\delta-1$.

4. If there exists an $(n, w-\delta, w)_{q} M O A-C W$ code, where $q-1 \leq \delta+1$, then $w \leq \delta+2$. 
Proof. All the claims are direct consequences of Theorems 5, 6, and 7, where the length $n$ in the $\mathrm{OA}(t, n, q)$ is restricted to $w$, the alphabet size is $q-1$, and $w-\delta=n-t+1$.

Theorem 22 implies upper bounds on $w$ as a function of the alphabet size $q$ and the minimum distance $d$ of the code and, similarly, lower bounds on $q$ as a function of the weight $w$ and the minimum distance of the code. Since $d=w-\delta$, it follows that these bounds can be written as bounds only as a tradeoff between $d$ and $q$.

\section{Corollary 15.}

1. If there exists an $(n, d, w)_{q} M O A-C W$ code, where $1 \leq d \leq w-2$ and $q$ is even, then $d \leq q$.

2. If there exists an $(n, d, w)_{q} M O A-C W$ code, where $1 \leq d \leq w-2$ and $q$ is odd, then $d+1 \leq q$.

The next bound presents a tradeoff between the length, the weight, and the alphabet size, of the code. It is interesting to note that the minimum distance has no influence on the bound.

Theorem 23. If there exists an $(n, d, w)_{q} M O A-C W$ code, then $n \leq q+w-2$ (equivalently, $q \geq n-w+2)$.

Proof. Let $\mathcal{C}$ be an $(n, d, w)_{q}$ MOA-CW code and consider the set $\mathcal{S}$ of codewords in $\mathcal{C}$ which have only nonzero symbols in the first $w-1$ coordinates and in these $w-1$ coordinates of $\mathcal{S}$, all the codewords of $\mathcal{S}$ share the same suffix of length $w-d+1$. Each two such codewords of $\mathcal{S}$ can differ in at most two coordinates out of the last $n-w+1$ coordinates and in the first $d-2$ coordinates. Hence, since the minimum distance of $\mathcal{C}$ is $d$, it follows that two such codewords of $\mathcal{S}$ differ exactly in these $d$ coordinates. This implies the following observations:

1. The only nonzero symbol in the last $n-w+1$ coordinates of each codeword from $\mathcal{S}$ is in a distinct coordinate. This implies that $|S| \leq n-w+1$. Moreover, since the projection, on the nonzero entries, of the codewords whose support is a given subset of $w$ coordinates forms an $\mathrm{OA}(w-d+1, w, q-1)$, it follows that $\mathcal{S}$ contains a codeword with a nonzero symbol in each one of the last $n-w+1$ coordinates and hence $|S| \geq n-w+1$. Thus, $|S|=n-w+1$.

2. Each two codewords of $\mathcal{S}$ differ in all the symbols of their first $d-2$ coordinates. This implies that $q-1 \geq|S|$.

Thus, $q-1 \geq n-w+1$, which completes the proof of the theorem.

\subsection{Comparison between maximum size anticodes}

So far we have characterized the families of diameter perfect constant-weight codes. Each family is associated with some maximum size anticodes. In this subsection we will characterize these families of maximum size anticodes and compare some of them. 
The first family of maximum size anticodes is associated with the family [[F1]] of nonbinary diameter perfect constant-weight codes for which $w=n$. Clearly, for these anticodes the length of a codeword is $n$ and the weight of each codeword is $w=n$. Moreover, the anticodes are derived from the related anticodes in the Hamming scheme, by replacing the zeroes in the anticodes of the Hamming scheme with the additional nonzero symbol of the constant-weight code.

The second family of maximum size anticodes is associated with the family $[[\mathbf{F} 2]]$ of diameter perfect constant-weight codes over an alphabet of size $2^{k}+1$ for which $w=n-1$. Clearly, the related anticodes also have length $n$ and weight $w=n-1$. If the non-binary diameter perfect code is in fact a non-binary perfect code, then the related anticode is a ball. If the non-binary diameter perfect code is not a non-binary perfect code, then the related anticode is not a ball and it should be computed for each set of parameters. For example, it was proved in [25] that for ternary codes if $n=2^{m} \geq 8, w=n-1$, an the diameter is 4 , then the maximum size anticode has size $n^{2}$ and such a set can be defined by the union of the set of ternary words with a unique zero and all the other symbols are ones and the set of ternary words with a unique zero and a unique two and all the other symbols are ones.

The third family of maximum size anticodes is associated with the family of generalized Steiner system. The related anticode $\mathcal{A}^{\mathrm{s}}(n, w, t)$ was defined by

$$
\mathcal{A}^{\mathrm{s}}(n, w, t) \triangleq\left\{(\overbrace{1 \cdots \cdots 1}^{t \text { times }}, a_{1}, \ldots, a_{n-t}): a_{i} \in \mathbb{Z}_{q}, \operatorname{wt}\left(a_{1} \cdots a_{n-t}\right)=w-t\right\} .
$$

This anticode has diameter $2(w-t)$ (when $n-t \geq 2(w-t))$ and its size is $\left(\begin{array}{c}n-t \\ w-t\end{array}\right)(q-1)^{w-t}$.

The last family of maximum size anticodes is associated with families $[\mathbf{F} 4],[\mathbf{F 5}]$, and $[\mathbf{F 6}]$ of the diameter perfect constant-weight codes. The related anticode $\mathcal{A}^{\mathrm{m}}(n, w, \delta)$ was defined by

$$
\mathcal{A}^{\mathrm{m}}(n, w, \delta) \triangleq\left\{(a_{1}, \ldots, a_{\delta}, \overbrace{1 \cdots \cdots 1}^{w-\delta \text { times }}, \overbrace{0 \cdots \cdots 0}^{n-w}): a_{i} \in \mathbb{Z}_{q} \backslash\{0\}\right\},
$$

This anticode has diameter $\delta$ and its size is $(q-1)^{\delta}$.

One can be easily observed that nontrivial anticodes of the first two families cannot have the same parameters since they have different weights. Moreover, it can be observed that nontrivial anticodes from these two families cannot have the same parameters as the anticodes from the last two families. Hence, we will compare the anticodes from the last two families.

Unless the two anticodes represent one of two trivial cases $(w=n$ and $\delta=w-t$; or $w=t)$ they cannot be isomorphic. This can be observed from the fact that the zeroes of $\mathcal{A}^{\mathrm{m}}(n, w, \delta)$ are in $n-w$ fixed coordinates, while the zeroes of $\mathcal{A}^{\mathrm{s}}(n, w, t)$ are in any combination of $n-w$ coordinates in the last $n-t$ coordinates.

Can these two anticodes be maximum size anticodes, related to two diameter perfect codes of different families, be of the same size (when the length, weight, and diameter are the same)? Note first that this implies that the related code from the family [F4], or the family [F5], or the family $[\mathbf{F 6}]$ must be also a generalized Steiner system since the two codes will have the same parameters. Since $\left|\mathcal{A}^{\mathrm{s}}(n, w, t)\right|=\left(\begin{array}{c}n-t \\ w-t\end{array}\right)(q-1)^{w-t}$ and $\left|\mathcal{A}^{\mathrm{m}}(n, w, \delta)\right|=(q-1)^{\delta}$, it follows that the two anticodes are of equal size if and only if $\left(\begin{array}{c}n-t \\ w-t\end{array}\right)=(q-1)^{\ell}$ for some nonnegative integer $\ell=\delta+t-w$. If $\ell=0$, then either $n=w$ (the first trivial case) or $w=t$ 
(the second trivial case). We distinguish now between two cases depending on whether $\ell=1$ or $\ell>1$.

1. If $\ell=1$, then $\left(\begin{array}{c}n-t \\ w-t\end{array}\right)=q-1$ and we distinguish between three cases, depending on whether the related code is from the family $[\mathbf{F} 4]$, the family $[\mathbf{F 5}]$, or the family $[\mathbf{F 6}]$.

Case 1.1. The diameter perfect code is from the family $[\mathbf{F 4}]$ which implies that $\delta=w$ and hence $t=\ell=1$.

Since $t=1$, it follows that $\left(\begin{array}{l}n-1 \\ w-1\end{array}\right)=q-1$ and hence by Corollary 8 , there exists an $(n, w+1, w)_{q}$ code of the family [F4]. If $w=2$, then $q=n$ and the two related codes are a GS $(1,2, n, n)$ which is also an $(n, 3,2)_{n}$ code from the family [F4]. Such a code exists by Corollary 11. If $2<w<n$, then a related code with $\left(\begin{array}{l}n \\ w\end{array}\right)$ codewords cannot be a $\operatorname{GS}(1, w, n, q)$.

Case 1.2. The diameter perfect code is from the family $[\mathbf{F 5}]$ which implies that $\delta=w-1$ and hence $t=\ell+1=2$.

For the $\operatorname{GS}(2, w, n, q)$ and the $(n, w, q)$ MDS-CW code to be equal they must have the same minimum distance and hence $2(w-2)+1=w$, i.e., $w=3$. Since also $\left(\begin{array}{l}n-2 \\ w-2\end{array}\right)=q-1$, it follows that $n=q+1$. Two codes are considered in this case. The first one is a generalized Steiner system $\operatorname{GS}(2,3, q+1, q)$ derived from a 1-perfect Hamming code over $\mathbb{F}_{q}$. The second one is an $(q+1,3, q)$ MDS-CW code derived from a $[q+1, q-1,3]_{q}$ MDS code. For these parameters the 1-perfect Hamming code is also an MDS code and hence both constant-weight codes are the same code. There might be other such constant-weight codes for $q$ which is not a power of a prime, but no such code is known.

Case 1.3. The diameter perfect code is from the family $[\mathbf{F 6}$ ], for which the MOA-CW code has minimum Hamming distance $d<w$, which implies that $\delta=d-1$ and hence $t=\ell+1+w-d=w-d+2$.

Hence, the related codes are $\mathrm{GS}(t, w, n, q)$ and an $(n, d, w)_{q} \mathrm{MOA}-\mathrm{CW}$ code. The codes have the same minimum Hamming distance and hence $d=2(w-t)+1=2 d-3$, i.e., $d=3$, which implies that $w=t+1$. Since $\left(\begin{array}{l}n-t \\ w-t\end{array}\right)=q-1$, it follows that $n-t=q-1$, i.e., $n=q+t-1$, and hence one of our codes is a $\operatorname{GS}(t, t+1, q+t-1, q)$. By iteratively applying Lemma 9, we obtain a $\mathrm{GS}(2,3, q+1, q)$ which is the code in the previous case. Unfortunately, no $\mathrm{GS}(t, t+1, q+t-1, q)$ is known for $t>2$.

2. If $\ell>1$, then $\left(\begin{array}{c}n-t \\ w-t\end{array}\right)=(q-1)^{\ell}$ and first we have to consider the solutions for this equation. We distinguish between three cases depending whether $w-t \in\{1, n-t-1\}$, $w-t \in\{2,3, n-t-3, n-t-2\}$, or $3<w-t<n-t-3$.

Case 2.1. If $w-t \in\{1, n-t-1\}$.

If $w-t=n-t-1$, then $w=n-1$ and one code is a $\operatorname{GS}(t, n-1, n, q)$ and by iteratively applying Lemma 9, we obtain a $\mathrm{GS}(1, n-t, n-t+1, q)$. One can easily verify that for such a code $n-t+1 \geq 1+(n-t+1)(q-1)$ (see also [16]) and hence it does not exist.

If $w-t=1$, then one code is a $\mathrm{GS}(t, t+1, n, q)$ for which the minimum distance is 3 . Hence, the related codes from the families $[\mathbf{F 4}],[\mathbf{F 5}]$, and $[\mathbf{F} \mathbf{6}]$ are only those 
considered in cases 1.1., 1.2., and 1.2., respectively. Therefore, no such code will be found for $\ell>1$.

Case 2.2. If $w-t \in\{2, n-t-2\}$.

When $w-t=2$ or $w-t=n-t-2$, there are infinitely many such solutions which satisfy the recursion $a_{m}=6 a_{m-1}-a_{m-2}$ (where $q-1=a_{m}$ and $\ell=2$ ) [Online Encyclopedia on Integer Sequences A001109], with the initial conditions $a_{1}=1$ and $a_{2}=6$. Similar analysis to the previous cases shows that there is no diameter perfect code from two different families in this case.

Case 2.3. If $2<w-t<n-t-2$.

It was proved by Erdös [26, p. 48] that this equation has exactly one solution for $n-t=50$ and $w-t=3$ or $w-t=n-t-3$. In the region for this solution there is no code from two families.

Therefore, all those cases for which the anticodes $\mathcal{A}^{\mathrm{s}}(n, w, t)$ and $\mathcal{A}^{\mathrm{m}}(n, w, \delta)$ have the same size and different structure, the anticodes are not related to a diameter perfect codes from two different families. Moreover, they might not be of maximum size.

In general, one can decide based on the size of a maximum size anticode if the given parameters are in the range of a generalized Steiner system, an MDS-CW code, or an MOA-CW code. Each such code is an optimal non-binary constant-weight code that meets the value of $A_{q}(n, d, w)$. In some cases these codes coincide as illustrated in the following example (and analyzed in the comparison of the codes with their anticodes).

Example 1. Let $\mathcal{C}$ be a linear 1-perfect code of length $q+1$, dimension $q-1$, and minimum Hamming distance 3, over $\mathbb{F}_{q}$. By its parameters, this code is also an MDS code. The codewords of weight three of $\mathcal{C}$ form a generalized Steiner system $G S(2,3, q+1, q)$ and also $a(q+1,3, q)$ MDS-CW code. The related maximum size anticodes are $\mathcal{A}^{s}(q+1,3,2)$ and $\mathcal{A}^{m}(q+1,3,1)$ which are of the same size $(q-1)^{2}$, but different structure.

\section{Conclusions and Open Problems for Future Research}

We have considered diameter perfect constant-weight codes. First, we have revisited the family of such binary constant-weight codes which are codes in the Johnson scheme. Nonbinary such codes (where the metric is the Hamming distance) are not associated with an association scheme and hence the original proofs for the code-anticode bound do not hold for these codes. Also proofs which were given to other spaces with related metrics, which do not require the association scheme conditions, do not hold for these codes. We have presented a novel proof to the code-anticode bound for these codes. We have distinguished between six families of such codes and four families of related maximum size anticodes. All the new constructed non-binary diameter perfect constant-weight codes are optimal codes, which attain the value of $A_{q}(n, d, w)$. Two of the families of anticodes are new and the proof of their optimality is a simple consequence from the code-anticode bound. As was pointed out in [1] maximum size anticodes in the Hamming scheme, the Johnson scheme, and the Grassmann scheme, are related to $t$-intersecting families and the celebrated Erdös-Ko-Rado theorem. The proofs that certain $t$-intersecting families (anticodes) are of maximum size are 
not simple and they were mainly obtained via extremal combinatorics. This is in contrast to our proofs which are very simple since they are derived from the code-anticode bound. Four of the families of diameter perfect constant-weight codes were considered before, but they were not observed as diameter perfect constant-weight codes. The last two families are new and raise many problems for future research. Some of these problems, as well as other problems related to the other families, will be presented now.

1. Are there diameter perfect codes in $\mathrm{J}(n, w)$, except for Steiner systems and their complements? By Conjecture 1 such codes do not exist, but there are hardly any result in this direction. It was proved in [1] (who used an idea from [15]) that if such a diameter perfect code exists then some Steiner systems also exist. This excludes the existence of some parameters of such diameter perfect codes as a consequence of the necessary conditions in Corollary 4.

2. Continue to develop the theory of generalized Steiner systems $\operatorname{GS}(t, w, n, q)$. Even for small parameters, such as $(t, w)=(2,3)$, we don't know if the necessary conditions similar to the ones in Corollary 4 are sufficient, although there are many results on these parameters, e.g. [10, 16, 29]. We conjecture that this is the case for all $q$, with a possible exceptions for a small number of values of $n$. For other parameters there are also lot of research work, e.g. [7, 16, 35], but there is no known construction for $t>3$ and we would like to see a construction for such a system.

3. For a given pair $(w, n), 3 \leq w<n$, find good upper bound on the alphabet size $\operatorname{QMDS}(w, n)$, for which there exist an $(n, w, q) \operatorname{MDS}-\mathrm{CW}$ code for each $q \geq \operatorname{QMDS}(w, n)$. It was proved in [16] that for each pair $(w, n)$ there exists such a value $\operatorname{QMDS}(w, n)$, but the current upper bound is very large.

4. For any given pair $(w, n), 4 \leq w \leq n-2$, improve the upper bound on $q=q_{0}(w, n)$, which is the smallest alphabet for which there exist an $(n, w+1, w)_{q}$ code. A very intriguing problem is this direction is to find good lower bounds and upper bounds on $\chi(w, n)$, the minimum number of codes in a partition of all binary words of length $n$ and weight $w$ into codes with minimum Hamming distance $w+2$.

5. Present new constructions for MDS-CW codes (and also for MOA-CW codes). The only construction, which is not derived directly from an orthogonal array, which we gave was analyzed in Theorem 20. Another construction is the union construction for MDS-CW codes as mentioned in Theorem 15. Is there a related construction for MOA-CW codes? We would like to see new different constructions as well as amendments to the construction which was given in the paper.

6. Present new bounds on the tradeoff between the parameters of MDS-CW codes (and also for MOA-CW codes). This is especially important and we would like to see some new directions which will enable to conjecture for which parameters such codes exist.

7. Given $1<d<w<n$, does there exist a $q_{0}(n, d, w)$ for which there exists an $(n, d, w)_{q}$ MOA-CW for all $q \geq q_{0}(n, d, w)$ ? recall that for MDS-CW codes such a value called $\operatorname{QMDS}(w, n)$ exists as was proved in [16]. 
8. Are there more families of diameter perfect constant-weight codes, except for the six families which were presented? One possible direction, to exclude such possible families of codes, is to show sets of parameters with tradeoff between $n, w$, and $d$, where such codes cannot exist.

9. Characterize all parameters for which $\mathcal{A}^{\mathrm{s}}(n, w, t)$ is a maximum size anticode. Such a proof can be done by using extremal combinatorics as was done in [2] for such anticodes related to binary words with constant weight. Similarly, characterize all parameters for which $\mathcal{A}^{\mathrm{m}}(n, w, \delta)$ is a maximum size anticode. Such a proof can be also done by using extremal combinatorics as was done in [2] and in [3] for such anticodes related to binary words with constant weight and for non-binary words in the Hamming scheme. The range in which $\mathcal{A}^{\mathrm{m}}(n, w, \delta)$ is optimal can be proved by using a combination of techniques from both papers.

10. Are there $(q+1,3, q)$ MDS-CW codes which are also generalized Steiner systems $\mathrm{GS}(2,3, q+1, q)$ beside those for prime power $q$ ?

11. Is there any $\operatorname{GS}(3,4, q+2, q)$ for a prime power $q$ ? and for non-prime power $q$ ?

12. Define the anticodes $\mathcal{A}^{\mathrm{s}}(n, w, t)$ and $\mathcal{A}^{\mathrm{m}}(n, w, \delta)$ in terms of $t$-intersecting families. Find the maximum size of these $t$-intersecting families (in other words, the maximum size of these anticodes) for all parameters, including the cases where $d>w+1$.

\section{Acknowledgement}

The author would like to thank Denis Krotov for bringing [5, 24, 25] to his attention. He also would like to thank Daniella Bar-Lev for bringing [26] to his attention.

\section{References}

[1] R. Ahlswede, H. K. Aydinian, and L. H. Khachatrian, On perfect codes and related concepts, Designs, Codes, and Crypto. 22 (2001), 221-237.

[2] R. Ahlswede and L. H. Khachatrian, The complete intersection theorem for systems of finite sets, European J. Combin. 18 (1997), 125-136.

[3] R. Ahlswede and L. H. Khachatrian, The diametric theorem in Hamming spaces - optimal anticodes, Advances in Applied Math. 20 (1998), 429-449.

[4] A. E. Brouwer, James B. Shearer, N. J. A. Sloane, and Warren D. Smith, A new table of constant weight codes, IEEE Trans. Inf. Theory 36 (1990), 1334-1380.

[5] K. A. Browning, J. F. Dillon, M. T. McQuistan, and A. J. Wolfe, An APN permutation in dimension six, in: G. McGuire, G. L. Mullen, D. Panario, and I. E. Shparlinski, editors, Finite Fields: Theory and Applications. Proc. 9th Int'l Conf. on Finite Fields and Applications (Dublin, July 13-17, 2009), Contemporary Mathematics vol. 518, American Mathematical Society, Providence, RI, 2010, pp. 33-42. 
[6] S. Buzaglo and T. Etzion, Bounds on the size of permutation codes with the Kendall's $\tau$-metric, IEEE Trans. Inf. Theory 61 (2015), 3241-3250.

[7] H. CaO, L. Ji And L. Zhu, Constructions for generalized Steiner systems $G S(3,4, v, 2)$, Designs, Codes and Crypto. 45 (2007), 185-197.

[8] Y. M. Chee, S. H. Dau, A. C. H. Ling, and S. Ling, The sizes of optimal q-ary codes of weight three and distance four: a complete solution, IEEE Trans. Inf. Theory 54 (2008), 1291-1295.

[9] Y. M. Chee And S. Ling, (2007). Constructions for q-ary constant-weight codes, IEEE Trans. Inf. Theory 53 (2007), pp. 135-146.

[10] K. Chen, G. Ge, And L. Zhu, Generalized Steiner triple systems with group size five, J. of Combin. Designs 7 (1999), 441-452.

[11] C. J. Colbourn And J. H. Dinitz, Design Theory, Boca Raton, FL: Chapman and Hall/CRC Press, 2007.

[12] P. Delsarte, An algebraic approach to the association schemes of coding theory, Philips Res. Repts. Suppl. 10 (1973), 1-97.

[13] P. Delsarte, Pairs of vectors in the space of an association scheme, Report $R$ 325, MBLE Res. Lab., Brussels 1976.

[14] P. ERdös, C. Ko, And R. RAdo, Intersection theorems for systems of finite sets, Quart. J. Math. Oxford Ser. 12 (2001), 313-320.

[15] T. Etzion, On the nonexistence of perfect codes in the Johnson scheme, SIAM J. Discrete Math. 9 (1996), 201-209.

[16] T. Etzion, Optimal constant weight codes over $Z_{k}$ and generalized designs, Discrete Math. 169 (1997), 55-82.

[17] T. Etzion, Product constructions for perfect Lee codes, IEEE Trans. Inf. Theory 57 (2011), 7473-7481.

[18] T. Etzion, E. Gorla, A. Ravagnani, and A. Wachter-Zeh, Optimal Ferrers diagram rank-metric codes, IEEE Trans. Inf. Theory 62 (2016), 1616-1630.

[19] T. Etzion And van Lint, On perfect constant weight codes, Discrete Math. and Theoretical Computer Science on Codes and Association Schemes 56 (2001), 131-134.

[20] D. G. Fon-Der-FlaAss, A bound on Correlation immunity, Siberian Electron. Math. Rep. 4 (2007), 133-135.

[21] S. Glock, D. Kühn, A. Lo, And D. Osthus, The existence of designs via iterative absorption, arxiv.org/abs/1611.06827, January 2014.

[22] R. L. Graham and N. J. A. Sloane, Lower bounds for constant weight codes, IEEE Trans. Inf. Theory 26 (1980), 37-43. 
[23] P. Keevash, The existence of designs, arxiv.org/abs/1401.3665, January 2014.

[24] D. S. Krotov, On diameter perfect constant-weight ternary codes, Discrete Math. 308 (2008), 3104-3114.

[25] D. S. Krotov, P. R. J. Östergård, and O. Pottonen, Non-existence of a ternary constant weight $(16,5,15 ; 2048)$ diameter perfect code, Advances in Math. of Commun. 10 (2016), 393-399.

[26] F. Le Lionnais, Les nombres remarquables, Paris: Hermann, 1983.

[27] F. J. MacWilliams and N. J. A. Sloane, The Theory of Error-Correcting Codes, Amsterdam: North-Holland, 1977.

[28] P. R. J. Östergånd And M. Svanström, Ternary constant weight codes, Electron. J. Combin. 9 (2002), R41.

[29] K. Phelps And C. Yin, Generalized Steiner systems with block size three and group size $g \equiv 3(\bmod 6)$, J. of Combin. Designs 5 (1997), 417-432.

[30] D. Raghavarao, Constructions and Combinatorial Problems in the Design of Experiments, John Wiley: New York, 1971.

[31] C. Roos, Some remarks on perfect subsets in distance regular graphs, Delft Progr. Rep. 7 (1982), 90-97.

[32] M. Svanström, A class of perfect ternary constant-weight codes, Designs, Codes and Crypto. 18 (1999), 223-229.

[33] I. Tamo and M. Schwartz, Correcting limited-magnitude errors in the rankmodulation scheme, IEEE Trans. Inf. Theory 56 (2010), 2551-2560.

[34] J. H. van Lint and L. Tolhuizen, On perfect ternary constant weight codes, Designs, Codes and Crypto. 18 (1999), 231-234.

[35] D. Wu AND P. FAn, Construction of optimal quaternary constant weight codes via group divisible designs, Discrete Math. 309 (2009), 6009-6013.

[36] H. Zhang and G. Ge, Optimal quaternary constant-weight codes with weight four and distance five, IEEE Trans. Inf. Theory 59 (2013), 1617-1629. 